\title{
Osteoclasts secrete leukemia inhibitory factor to promote abnormal bone remodeling of subchondral bone in osteoarthritis
}

\author{
Xin Zhao 1,2, Long Ma , Haohui Guo', Jian Wang², Shuai Zhang ${ }^{1}$, Xiaochun Yang ${ }^{1}$, Lvlin Yang ${ }^{2}$ and Qunhua Jin ${ }^{1 *}$
}

\begin{abstract}
Background: Osteoarthritis $(\mathrm{OA})$ is a common chronic degenerative joint disease. At present, there is no effective treatment to check the progression of osteoarthritis. Osteochondral units are considered to be one of the most important structures affecting the occurrence and development of osteoarthritis. Osteoclasts mediate an increase in abnormal bone remodeling in subchondral bone in the early stage of osteoarthritis. Here, alendronate (ALN) that inhibit osteoclasts was used to study the regulatory effect of osteoclast-derived leukemia inhibitory factor (LIF) on early abnormal bone remodeling.
\end{abstract}

Methods: This study involved 10-week-old wild-type female C57BL/6 mice and female SOST knockout (KO) mice that were divided into the sham, vehicle, ALN, and SOST KO groups.

Results: The expression of LIF was found to decrease by inhibiting osteoclasts, and the histological OA score suggested that the degeneration of articular cartilage was attenuated. Additionally, micro-CT showed that osteoclasts inhibited in the early stage of OA could maintain the microstructure of the subchondral bone. The parameters of bone volume fraction (BV/TV), subchondral bone plate thickness (SBP.Th), and trabecular separation (Tb.Sp) of the treated group were better than those of the vehicle group.

Conclusions: These results suggested that downregulating the expression of sclerostin in osteocytes by secreting LIF from osteoclasts, activate the Wnt/ $\beta$-catenin signaling pathway, and promote abnormal bone remodeling in OA. Therefore, clastokine LIF might be a potential molecular target to promote abnormal bone remodeling in early OA.

Keywords: Osteoarthritis, Osteoclasts, Leukemia inhibitory factor, Alendronate, Sclerostin, Abnormal bone remodeling

\section{Introduction}

Osteoarthritis is a degenerative disease affecting the entire joint in individuals undergoing aging. Assessment of health-related quality of life (HRQOL) in the Chinese population shows that the severity of knee osteoarthritis affects the physical and mental health of patients [1]. The latest report from the United States bone and joint

*Correspondence: jinqunhua2020@163.com

1 Department of Orthopedics, General Hospital of Ningxia Medica University, 804 Shengli Street, Yinchuan, Ningxia, China

Full list of author information is available at the end of the article initiative show that the prevalence rate of $\mathrm{OA}$ in the past 3 months was $10.4 \%$, which is one of the most common diseases incurring the all-cause medical cost as high as 37.3 billion US dollars [2]. Joint injuries and deformities caused by this disease seriously affect the quality of life of the patients and bring a huge burden to families, government health expenditure, and the social economy. However, at present, the pathogenesis of osteoarthritis is still unclear, and there are no effective treatment measures. Therefore, it is necessary to clarify the pathogenesis of the disease to identify the prevention and treatment methods. original author(s) and the source, provide a link to the Creative Commons licence, and indicate if changes were made. The images or other third party material in this article are included in the article's Creative Commons licence, unless indicated otherwise in a credit line to the material. If material is not included in the article's Creative Commons licence and your intended use is not permitted by statutory regulation or exceeds the permitted use, you will need to obtain permission directly from the copyright holder. To view a copy of this licence, visit http://creativecommons.org/licenses/by/4.0/. The Creative Commons Public Domain Dedication waiver (http://creativeco mmons.org/publicdomain/zero/1.0/) applies to the data made available in this article, unless otherwise stated in a credit line to the data. 
To date, the origin of osteoarthritis whether from the cartilage or subchondral bone is controversial. Traditionally, osteoarthritis begins with the wear and tear of articular cartilage, but recent evidence suggests that subchondral bone remodeling is essential for initiating and leading to the disease progression [3]. The subchondral bone with mechanical support and the bone-cartilage interface with important mechanical properties are mildly damaged due to factors such as abnormal stress. This results in bone remodeling changes and then affects the integrity of the overlying cartilage [4]. The pathological changes in the subchondral bone are more obvious than those of cartilage in the early stage of OA [5].

Bone remodeling is regulated by the interaction between osteoblasts and osteoclasts at the site of injury and signal transduction releasing soluble mediators [6]. The imbalance of coupling bone remodeling is mediated by osteoclasts and osteoblasts. In addition, the migration of $\mathrm{T}$ and $\mathrm{B}$ cells to the injured site may be involved in osteoclastogenesis and bone catabolism [7]. In the early stage of OA, the activity of osteoclasts and osteoblasts increases by 190 and $96 \%$, respectively [3], which increase bone remodeling and bone loss. With the progress of the disease, the proliferation of osteoblasts and bone remodeling slows down, and OA progression aggravates [5].

LIF is a multifunctional secretory cytokine that participates in bone metabolism [8]. Grimandet al. reported that LIF is not expressed in osteoclasts under physiological conditions [9]. However, Koide et al. reported that osteoclasts mainly secrete LIF, which can inhibit sclerostin and bone turnover [10]. Sclerostin is a negative regulator of Wnt/ $\beta$-catenin signaling, which is encoded by the SOST gene secreted by the osteocytes. The decrease in sclerostin can activate the Wnt signaling pathway and promote bone formation [11]. Genome-wide association studies (GWAS) indicate that the Wnt signaling pathway plays a key role in the progression of osteoarthritis [12]. Therefore, the activation of osteoclasts in the early stage of OA may be the initial factor in the pathogenesis of OA and abnormal bone remodeling of the subchondral bone.

Osteoclast differentiation is induced by macrophage colony-stimulating factor (M-CSF) and receptor activator for nuclear factor- $\mathrm{kB}$ ligand (RANKL) [13]. In addition, the osteocytes in bone matrix increase osteoclast production in response to the apoptotic signal from microfractures [14]. Bisphosphonates are commonly used drugs for treating osteoporosis and fracture prevention. They effectively inhibit bone turnover and bone resorption. Mohammad et al. reported that alendronate attenuates the progression of OA in mice [15]. Therefore, this study aimed to investigate the mechanism of attenuating OA progression in an anterior cruciate ligament transection (ACLT) mice model by intraperitoneal injection of alendronate to inhibit osteoclasts. This would provide evidence to support the effect of osteoclast-secreting LIF on the abnormal bone remodeling of the subchondral bone, so as to clarify the pathogenesis of OA and provide the basis for clinical treatment.

\section{Materials and methods \\ Mice used in the study}

10-week-old wild-type female C57BL/6 mice were purchased from the Vital River (Beijing, China) and the female $S O S T$ gene knockout mice were provided by the research group. These animals were raised in SPF animal center, $12 / 12 \mathrm{~h}$ day and night, relative temperature $24-26^{\circ} \mathrm{C}$, relative humidity $50-60 \%$. They were then divided into the sham, vehicle, ALN, and SOST KO groups ( $n=6$ in each group). Before the operation, $1 \%$ pentobarbital sodium $(1.0 \mathrm{ml} / 100 \mathrm{~g})$ was used for intraperitoneal injection anesthesia. The anterior cruciate ligament (ACL) of the right knee was cut through the medial parapatellar approach to establish the OA model. For the sham and SOST KO groups, only the ACL of the right knee joint was exposed without cutting off after anesthesia, and the joint capsule and skin were sutured separately. After the operation, the mice were placed in a constant-temperature electric blanket for monitoring until they recovered naturally. The general health status, pain, and infection of mice were monitored daily after the operation. On the second day after ACLT, alendronate sodium (product No. A4978; Sigma-Aldrich; Merck) was injected intraperitoneally twice a week $(1000 \mu \mathrm{g} / \mathrm{kg} /$ dose) or vehicle (phosphate-buffered saline) of the same volume. The wild-type mice in the sham, vehicle, and ALN groups were euthanized at 0,4 , and 8 weeks after operation. The mice in SOST KO group was euthanized at 4 weeks. All the animal experimental schemes were examined and approved by the medical research ethics committee of the General Hospital of Ningxia Medical University (Yinchuan, China) (protocol No. 2018-056).

\section{Histochemical study}

Following euthanasia, the right knee joint was collected and fixed with $4 \%$ paraformaldehyde for $24 \mathrm{~h}$. Then, the specimens were decalcified in EDTA decalcification solution for 4 weeks and then embedded in paraffin. The wax block was fixed on a tissue slicer. The medial compartment was sectioned continuously with a thickness of $4 \mu \mathrm{m}$ and was used for safranin-O fast green staining,and a histological score was obtained. Dewaxing with two changes of xylene for $10 \mathrm{~min}$. Treating with three changes of $100 \%$ absolute ethanol for $3 \mathrm{~min}, 95 \%$ ethanol for $3 \mathrm{~min}, 70 \%$ ethanol for $2 \mathrm{~min}$. Harris Hematoxylin was developed the treating slides for $3 \mathrm{~min}$, then the slides were washed in tap water for $10 \mathrm{~min}$. Sections 
were stained with $0.2 \%$ Fast Green (product No. F7258; Sigma-Aldrich; Merck) for $3 \mathrm{~min}$, and then washed by $1 \%$ acetic acid for $5 \mathrm{~s}, 0.1 \%$ Safranin O (product No. S8884; Sigma-Aldrich; Merck) for $3 \mathrm{~min}$. Sections were hydrated in $95 \%$ ethanol for $5 \mathrm{~s}, 100 \%$ ethanol for $15 \mathrm{~s}$, fixation by 2 changes in xylene. The Osteoarthritis Research Society International (OARSI) score was used to assess the histopathological grading of cartilage.

\section{The tartrate-resistant acid phosphatase staining}

The tartrate-resistant acid phosphatase (TRAP) staining was performed according to the standard protocol. After the sections were treated with $70 \%$ ethanol, the sections were covered with TRAP stain for $30 \mathrm{~min}$. TRAP buffer is produced by acetate anhydrous (product No. S818278; Macklin) $2.05 \mathrm{~g}$, tartrate tetrahydrate (product No. P816440; Macklin) $5.65 \mathrm{~g}$ and $\mathrm{RO}$ water $500 \mathrm{ml}(\mathrm{pH} 5.0$ ). TRAP stain is produced by TRAP buffer $50 \mathrm{ml}$, Naphthol AS-MX Phosphate (product No. N4875; Sigma-Aldrich; Merck) $5 \mathrm{mg}, \mathrm{N}, \mathrm{N}$-Dimethylformamide (product No. D4551; Sigma-Aldrich; Merck) $0.5 \mathrm{ml}$ and Fast Red Violet LB Salt (product No. F3381; Sigma-Aldrich; Merck) $20 \mathrm{mg}$. Harris Hematoxylin was Developped the treating sections for $3 \mathrm{~min}$, then the sections were washed in tap water for $10 \mathrm{~min}$. The slides were dried overnight at $37^{\circ} \mathrm{C}$.

\section{Immunohistochemical, immunofluorescence, and histomorphometric studies}

A standard protocol was used for immunohistochemical staining. Sections were incubated with primary antibody, matrix metalloproteinase 13 (MMP-13) (ab39012, Abcam, 1:400), type $\mathrm{X}$ collagen (Col X, ab58632, Abcam, 1:100), LIF (ab138002, Abcam, 1:200), sclerostin (ab63097, Abcam, 1:50), $\beta$-catenin (ab16051, Abcam, 1:200), and osteocalcin (ab93876, Abcam, 1:200), overnight at $4{ }^{\circ} \mathrm{C}$. Subsequently, the polymer detection system (ZSGB BIO) was used to detect the immune activity, and then hematoxylin (ZSGB BIO) was used for counterstaining. For immunofluorescence staining, sections were incubated with Alexa Fluor ${ }^{\circledR} 488$ goat anti-rabbit secondary antibody (ab150077, Abcam, 1:500) for $1 \mathrm{~h}$ at $37^{\circ} \mathrm{C}$ in the dark. The morphological measurement of the subchondral bone tissue was performed using a Leica microscope (MD3000), the cartilage was evaluated by Zeiss fluorescence microscope (AXIO OBSERVERA1), and the quantitative analysis was performed blindly. For MMP-13 and $\mathrm{Col} \mathrm{X}$, the positive staining number and the total number of chondrocytes in the entire articular cartilage were calculated. For TRAP-positive osteoclasts, LIF, sclerostin, $\beta$-catenin, and osteocalcin in subchondral bone, three visual fields of each sample were taken for positive cell count and bone surface length measurement for analysis.

\section{Micro-computed tomography (Micro-CT) analysis}

Excess muscle tissues were removed from the entire right knee joint of the mice and fixed in 10\% formalin overnight. The specimens were scanned using a microcomputer (Inveon PET.SPECT.CT, Siemens). The images were scanned (Inveon Acquisition Workplace), reconstructed (COBRA_Exxim, Siemens), and further analyzed using the calculation software (Ineon Research Workplace). The X-ray current and voltage were set to $500 \mu \mathrm{A}$ and $80 \mathrm{kV}$, respectively, and the resolution was $8.5 \mu \mathrm{m} / \mu \mathrm{m} /$ pixel. The sagittal view of the entire medial compartment of the tibial subchondral bone was used for the 3D histomorphometry analysis, and the parameters of bone volume/total tissue volume (BV/TV), subchondral bone plate thickness (SBP.Th), and trabecular separation (Tb. Sp) were analyzed.

\section{Statistics}

The data are presented as mean $\pm \mathrm{SD}$. Independent sample $t$ test was used to determine the difference between the two groups, and one-way ANOVA was used to determine the differences between different groups. $P<0.05$ means the difference was statistically significant. All data were analyzed with IBM SPSS 19.0.

\section{Results}

Articular cartilage preservation by inhibiting osteoclasts First, the Safranin-O and Fast Green staining showed that the loss of proteoglycan in the corresponding ALN group was significantly reduced compared to that in the vehicle group 4 and 8 weeks after the operation (Fig. 1 A). The severity of cartilage degeneration was assessed using the OARSI histological score. After inhibition of osteoclast activity, the score of the ALN group was significantly lower than that of the vehicle group at 4 and 8 weeks after the operation (Fig. $1 \mathrm{~B}, \mathrm{C}$ ). The results of TRAP staining at 4 weeks confirmed that ALN could effectively inhibit osteoclasts (Fig. 1 D, E). Immunohistochemistry results showed that compared with the vehicle group, the number of MMP-13 positive cells in the ALN group decreased the injury of chondrocytes (Fig. 2 A, B). Col X data also showed similar results (Fig. 2 C, D). These results suggest that the inhibition of osteoclasts attenuated the degeneration of the articular cartilage.

\section{Maintenance of subchondral bone microstructure by inhibiting osteoclasts}

Micro-CT was used to further study the effect of ALN on the microstructure of subchondral bone and its correlation with articular cartilage protection. Inhibition of osteoclasts improved the microstructure of the subchondral bone. BV/TV in the vehicle group was significantly 


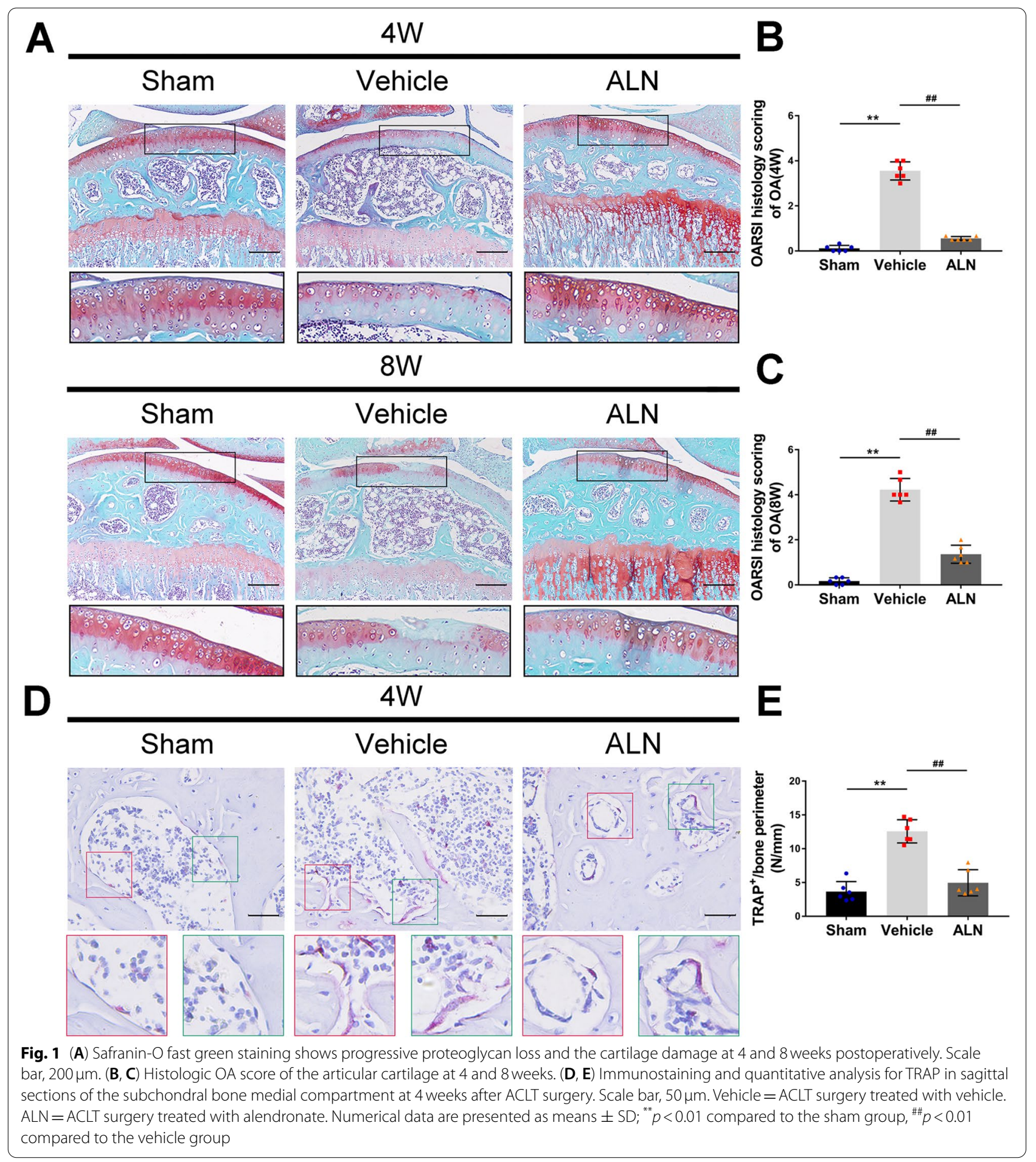

lower than that of the sham group 4weeks after the surgery, while the value in the ALN group was significantly higher. Similar results were obtained in the data 8 weeks after the surgery (Fig. 3 A, B). The subchondral bone plate closely related to articular cartilage decreased significantly in the vehicle group compared to the sham group at 4weeks post-operatively, while the ALN group maintained the thickness of the subchondral bone, which indicated that ALN eliminated the influence of bone resorption on the thickness of the subchondral bone 

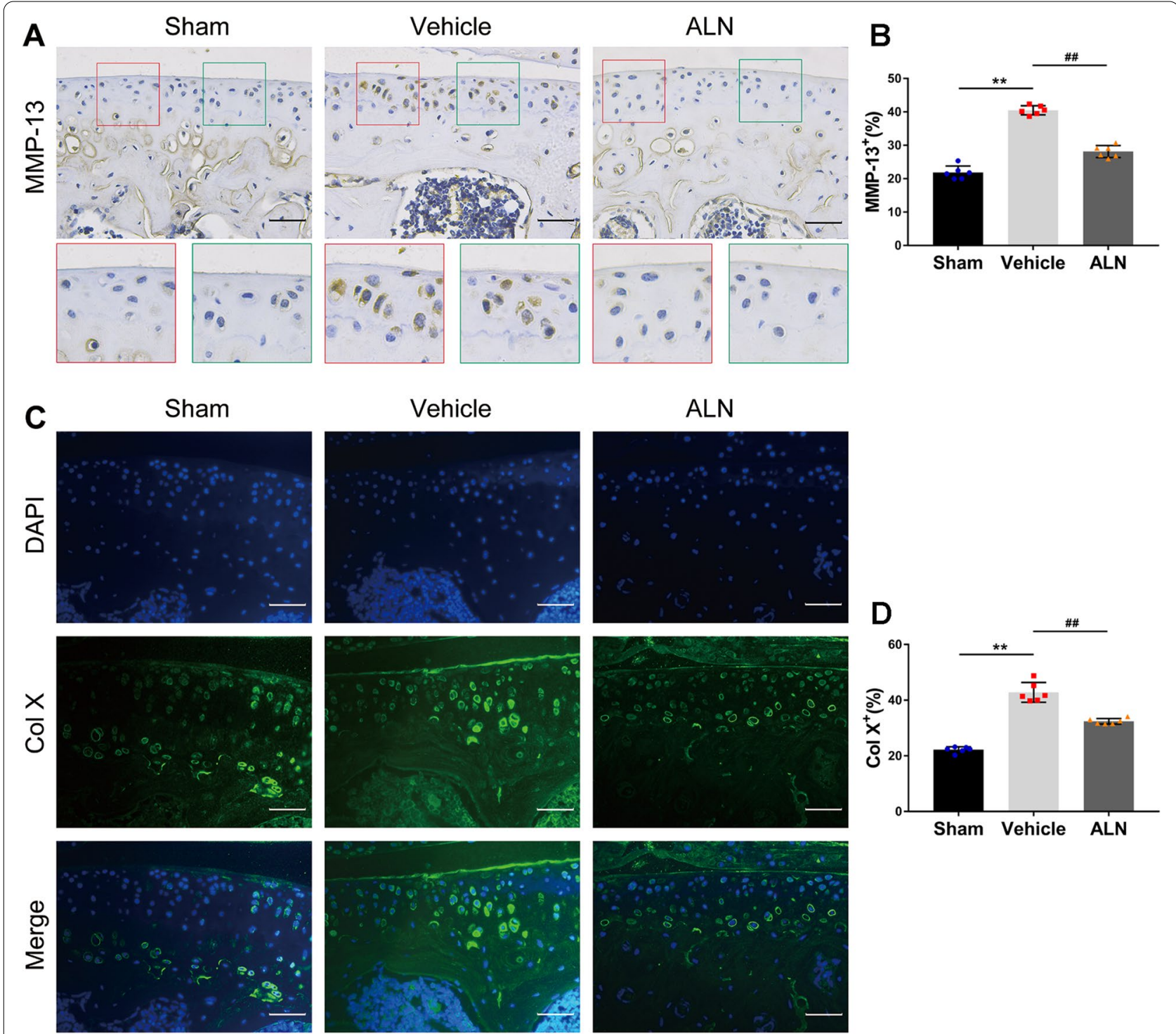

Sham Vehicle ALN

Fig. 2 (A, B) Inhibition of osteoclasts can reduce the ratio of matrix metalloproteinase-13 (MMP-13) positive cells in articular cartilage at 4 weeks after ACLT surgery. Immunostaining and quantitative analysis for MMP-13. (C, D) Immunofluorescence staining and quantitative analysis for collagen X. Scale bar, $50 \mu \mathrm{m}$. Means \pm SD; ${ }^{* *} p<0.01$ compared to the the sham group, ${ }^{\# \#} p<0.01$ compared to the the vehicle group

plate. Compared to the 4-week sham group, the values of the vehicle group decreased first and then increased at 4 , and 8 weeks, which showed that the subchondral bone plate changed from bone absorption to bone formation (Fig. $3 \mathrm{~A}, \mathrm{C}$ ). The increased Tb. Sp indicated that bone catabolism was greater than anabolism. Compared to the sham group at 4 and 8 weeks after surgery, the vehicle group increased significantly, and the ALN group abrogated the effect of modeling. (Fig. 3 A, D). These data indicated that the inhibition of osteoclasts by ALN could attenuate the process of abnormal bone remodeling of the subchondral bone after modeling.

\section{Effect of LIF secreted by osteoclasts on the abnormal bone} remodeling of the subchondral bone

Immunohistochemical staining was used to study the effect of clastokine LIF on the microstructure of the subchondral bone. Compared to the vehicle group at 0 weeks after the operation, the LIF positive cells of osteoclasts in the vehicle group increased significantly 4 weeks later but decreased after 8 weeks (Fig. 4 A, B). The TRAP staining showed similar results (Fig. 4 A, C). At 0, 4, and 8 weeks after the surgery, the number of sclerostinpositive cells in the vehicle group decreased gradually (Fig. 4 A, D), while the number of $\beta$-catenin-positive 


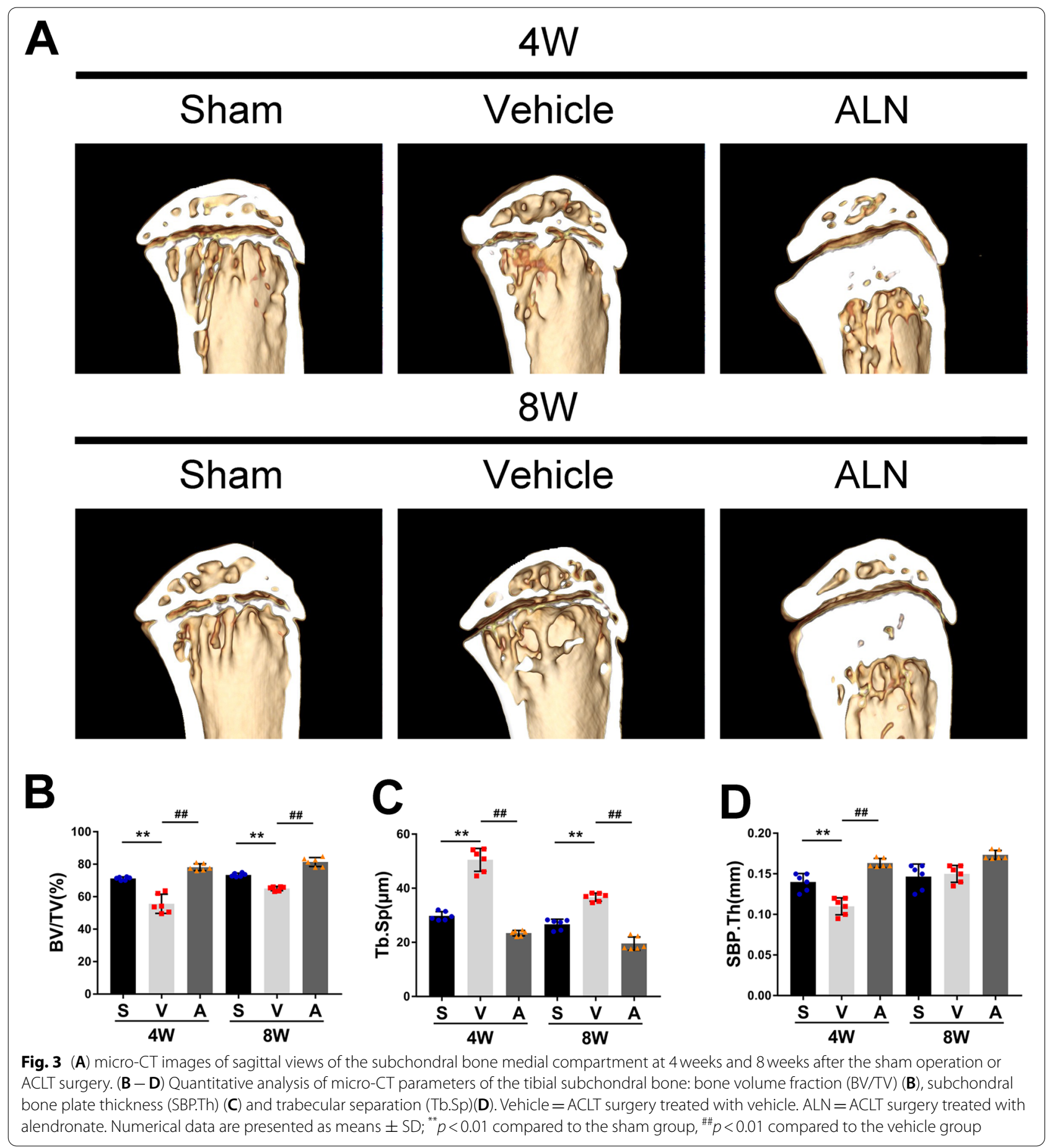

cells in osteoblasts increased gradually, which was similar to the results of osteocalcin (bone formation index) of osteoblasts (Fig. 3 A, E, F). Compared with the SOST KO group, the number of $\beta$-catenin-positive cells in the sham group was significantly higher than that in the SOST KO group, indicating that sclerostin negatively regulates the expression of $\beta$-catenin in the osteoblasts (Fig. $5 \mathrm{~A}-\mathrm{C}$ ). These results suggested that osteoclast activation and LIF expression might downregulate sclerostin, and then activate the $\beta$-catenin signal, which results in increased bone formation and slower bone remodeling. 

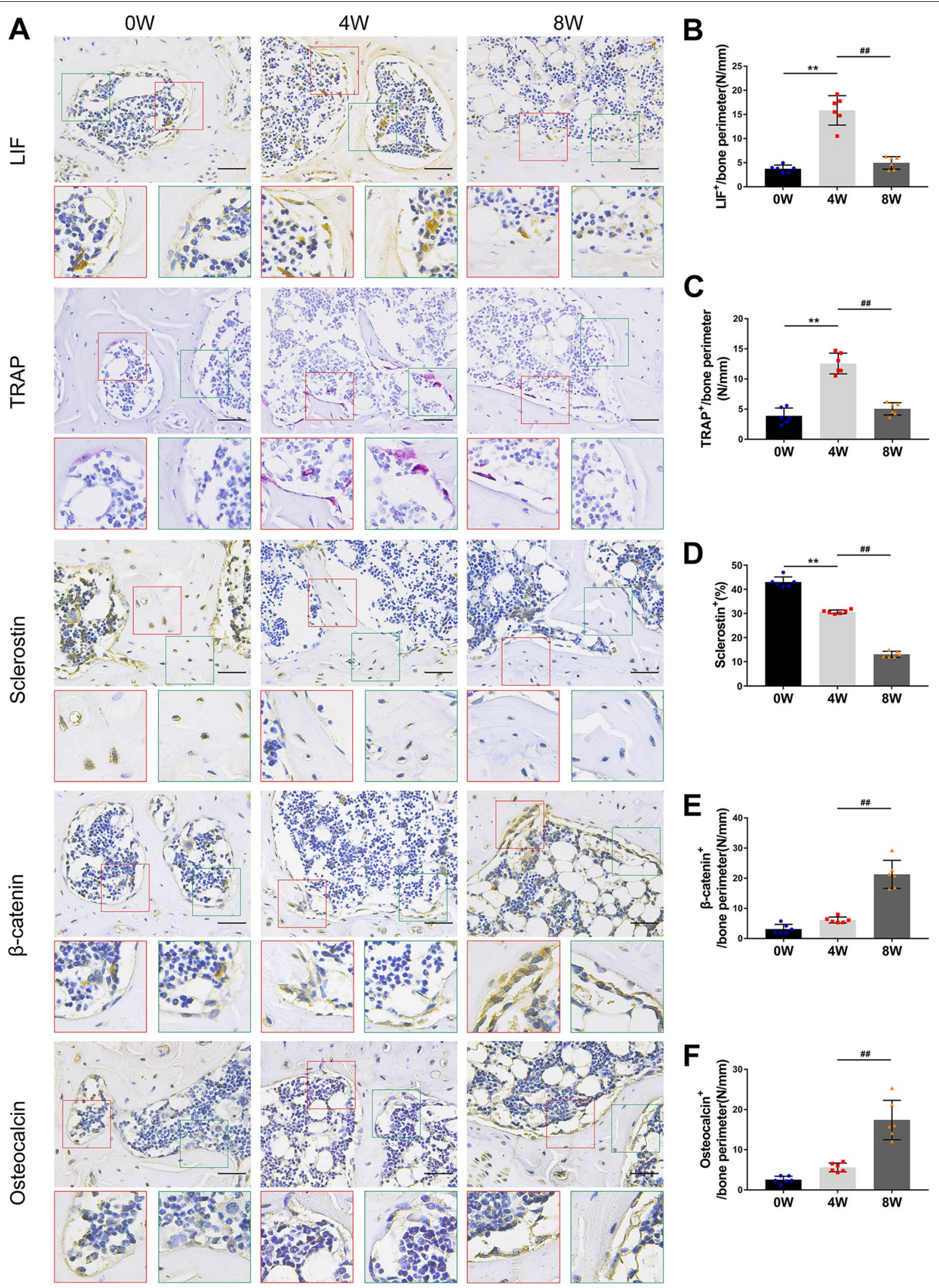

Fig. 4 (A - F) Immunostaining and quantitative analysis for $\operatorname{LIF}(\mathbf{A}, \mathbf{B}), \operatorname{TRAP}(\mathbf{A}, \mathbf{C})$, sclerostin $(\mathbf{A}, \mathbf{D}), \beta$-catenin $(\mathbf{A}, \mathbf{E})$ and osteocalcin $(\mathbf{A}, \mathbf{F})$ in sagittal sections of the subchondral bone medial compartment at 4 weeks and 8 weeks after ACLT surgery. Scale bar, $50 \mu \mathrm{m}$. Means \pm SD; ${ }^{* *} p<0.01$ compared to the vehicle group at 0 weeks, ${ }^{\# \#} p<0.01$ compared to the vehicle group at 4 weeks 


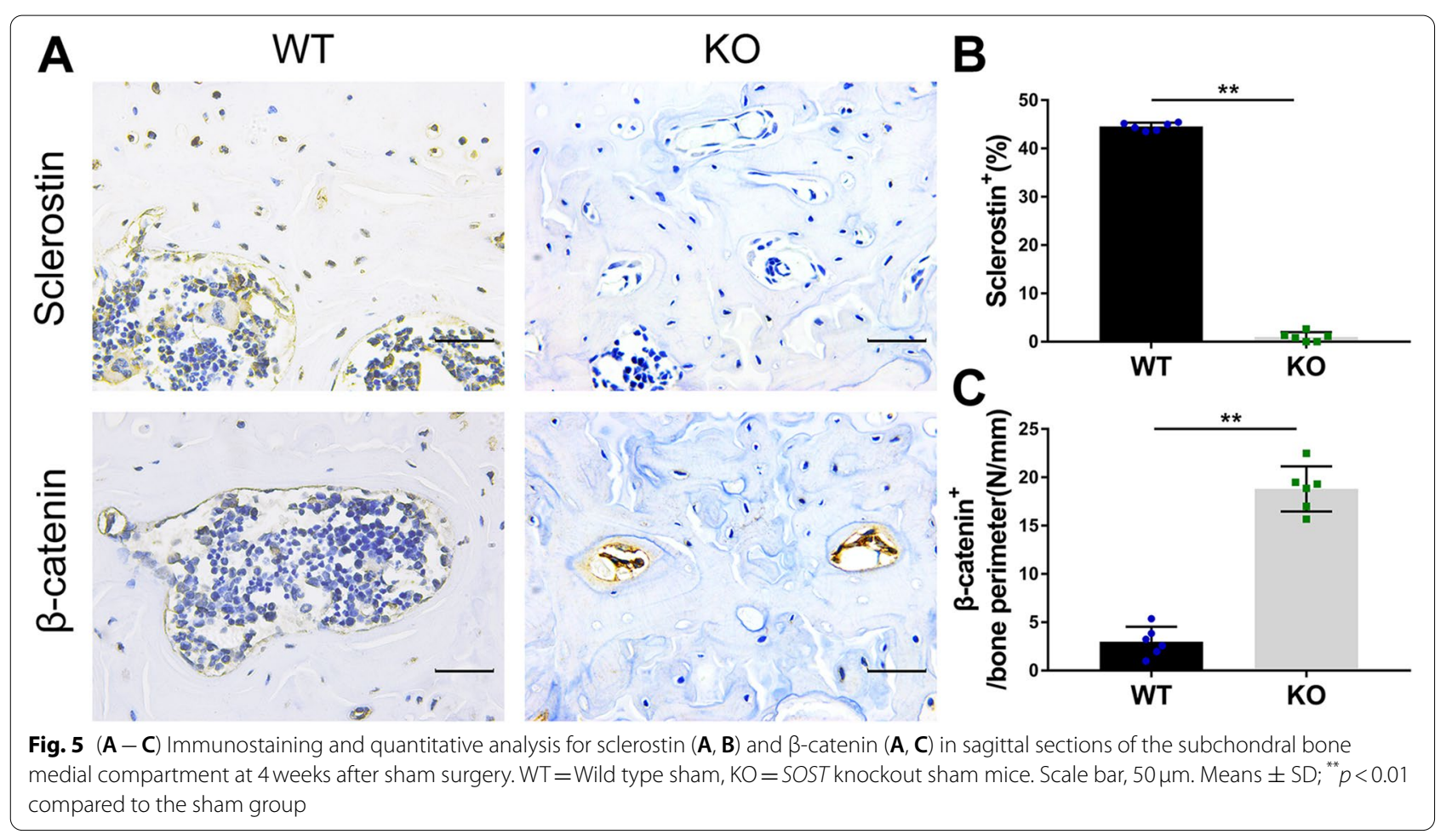

\section{ALN inhibited osteoclasts and attenuated abnormal bone remodeling of the subchondral bone}

Furthermore, the study investigated the effect of ALN on osteoclasts and found a decrease in LIF secretion on the microstructure of subchondral bone. The results showed that the number of LIF positive cells in the vehicle group was significantly higher than that in the sham group at 4 weeks after the operation, while the ALN group attenuated the difference (Fig. $6 \mathrm{~A}, \mathrm{~B}$ ). This was similar to the results of TRAP staining of osteoclasts (Fig. $6 \mathrm{~A}, \mathrm{C}$ ). However, the number of osteoclasts has affected the expression of sclerostin. At 4 weeks after the operation, sclerostin in the vehicle group was significantly decreased, while that in the ALN group was close to that in the sham group (Fig. $6 \mathrm{~A}, \mathrm{D}$ ). The number of $\beta$-catenin-positive cells in the vehicle group was higher than that in the sham group. Compared to $\beta$-catenin in the sham group, the bone formation ability was increased in the ALN group (Fig. $6 \mathrm{~A}, \mathrm{E}$ ). These results showed that by inhibiting the number of osteoclasts and reducing the expression of LIF, sclerostin could be restored, maintained the microstructure of the subchondral bone, and attenuated the process of abnormal bone remodeling.

\section{Discussion}

The progression of OA disease is known to be characterized by subchondral bone loss in the early stage and osteosclerosis in the late stage. A large amount of evidence suggests that subchondral bone remodeling plays a key role in the occurrence and development of OA [16]. This study demonstrated the potential mechanism of abnormal remodeling of the subchondral bone through the establishment of an unstable OA model and drug intervention. Specifically, We speculate that differentiated osteoclasts secrete a large amount of LIF, which inhibits the sclerostin in the surrounding osteocytes, and then activates the Wnt/ $\beta$-catenin pathway to change bone turnover, while ALN inhibits osteoclast activity, reducing the secretion of LIF, protecting the articular cartilage, and attenuating the process of $\mathrm{OA}$.

The microstructure of the subchondral bone changes with the development of OA. Research has shown that in the early stages of OA, young and middle-aged (2756 years old) patients have elevated subchondral bone resorption markers [17]. The increase in the bone resorption area elevates the risk of overlying cartilage damage [18]. MMP-13 can cause degradation of articular cartilage [19]. Col X is a specific marker of chondrocyte hypertrophy [20]. In this study, the ACLT model was used to increase the abnormal mechanical stress of the knee joint. The OARSI histology score, MMP-13, and Col $\mathrm{X}$ indicators in the vehicle group increased. At the same time, the micro-CT of the subchondral bone showed that compared to the sham group, the BV/TV, and SBP. Th of the vehicle group decreased, while $\mathrm{Tb}$. Sp increased. We found that when the basic cartilage structure was 


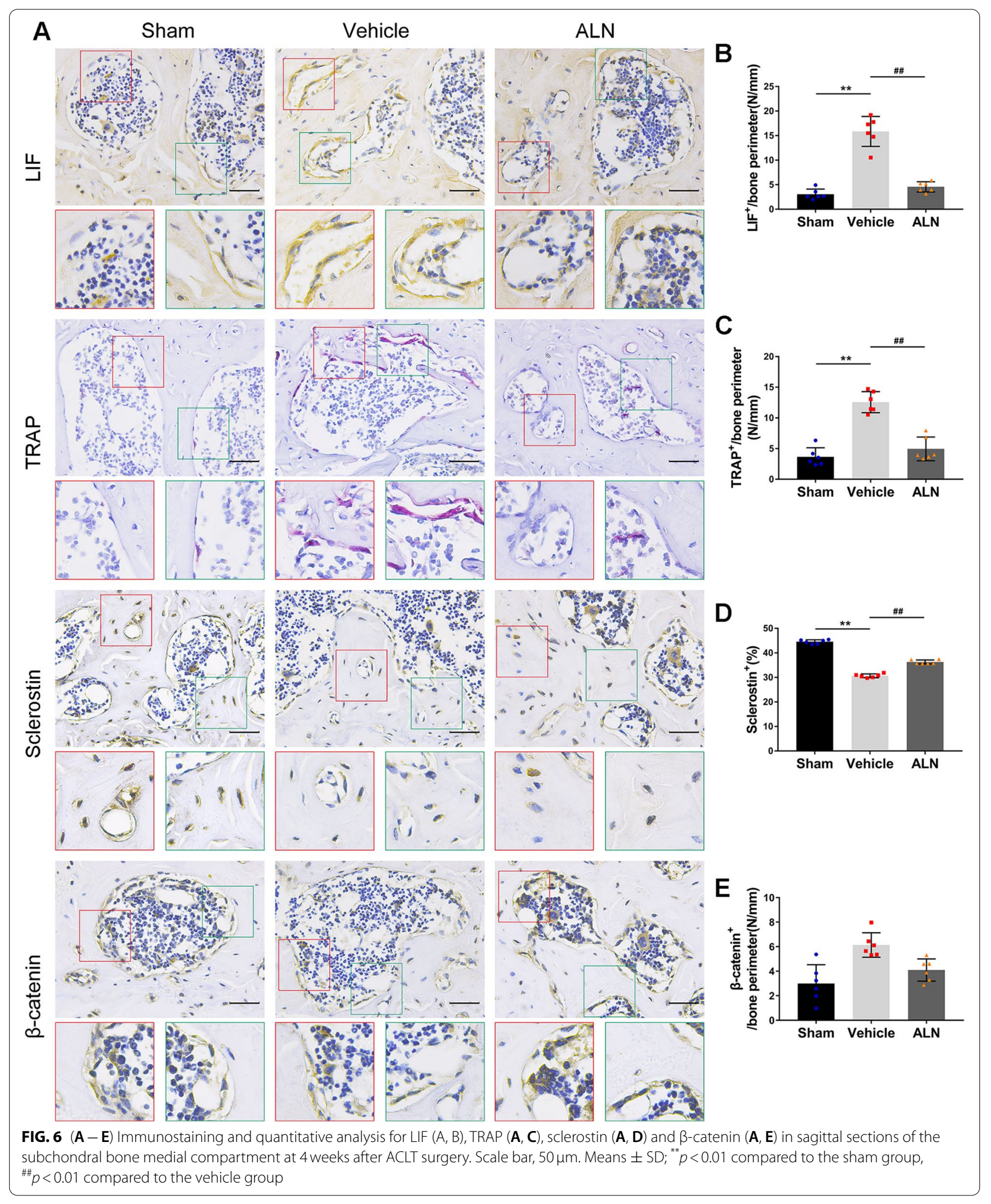


complete and there was no obvious damage, the microstructure of the subchondral bone changed to a large extent, mainly bone resorption. Compared to the vehicle group, the OARSI histological score, MMP-13, and Col $\mathrm{X}$ indicators were significantly improved after ALN treatment, and the micro-CT related parameters of subchondral bone were close to those of the sham group. These results indicate that the bone resorption of subchondral bone is increased in the early stage of OA, and the cartilage is progressively damaged. After inhibiting the activity of osteoclasts, the characteristics of subchondral bone are maintained, and the articular cartilage is protected.

The bone remodeling of subchondral bone mainly depends on the signal conduction between osteoclasts, osteoblasts, and osteocytes. The process involves bone resorption by osteoclasts on the surface of the bone and then bone formation. It is reported that the bone matrix releases transforming growth factor $\beta$ (TGF- $\beta$ ) and insulin-like growth factor I (IGF-I) during bone resorption, which induces the migration of osteoblast precursors to the site of bone resorption and promotes bone formation $[21,22]$. Previous studies have shown that TGF $-\beta 1$ can induce the expression of CXCL16 and leukemia inhibitory factor, and regulate the migration of osteoblast progenitor cells to recover bone loss during bone turnover and absorption [23]. Recent studies have shown that LIF secreted by mature and activated osteoclasts can promote bone formation by inhibiting the expression of sclerostin and upregulating Wnt/ $\beta$-catenin. LIF can bind to the cytokine binding region (CHR) of glycoprotein 130(gp130) on osteocytes to form the LIF/gp130/ LIFR complex and downregulate its sclerostin [24, 25]. In this study, the TRAP levels increased first and then decreased in the vehicle group 4 , and 8 weeks after the operation, which was consistent with the results of the subchondral bone turnover during OA progression. This was similar to the change in the LIF levels. The sclerostin level gradually decreased. After ALN intervention, the osteoclast activity was inhibited, corresponding to the decrease in LIF expression, and sclerostin was recovered. We speculate that the change in LIF may be have a potential effect in the turnover of subchondral bone abnormal remodeling in OA mice. In addition, other osteoclast-derived factors can also interact with LIFR to stimulate bone formation, such as cardiotrophin 1(CT-1) and oncostatin M (OSM). However, the results indicated that LIF, CT-1, and OSM might not be highly involved in inhibiting the expression of sclerostin in the state of high bone turnover [10].

Sclerostin binds to the extracellular domain of lowdensity lipoprotein receptor-related protein 5(LRP5) and antagonizes the Wnt signaling, which has a negative regulatory effect on the osteoblastic cells [26]. Our team has previously confirmed in human clinical samples that sclerostin negatively regulates $\beta$-catenin in the subchondral bone of OA patients [27]. The relationship between them was further proved by the SOST KO mice. In this study, the expression of $\beta$-catenin and osteocalcin increased gradually with the change of sclerostin in the vehicle group 4, and 8 weeks after the operation, and the abnormal bone remodeling of the subchondral bone transformed into bone formation, which was also supported by the micro-CT parameters. The deficiency of sclerostin can increase the osteogenic activity of subchondral bone and aggravate OA in mice [28]. This indicates that slow bone remodeling can accelerate the progression of $\mathrm{OA}$, while ALN can attenuate the turnover process.

ALN can eliminate the abnormal increase in bone remodeling in early OA. It reduces the number of osteoclasts and promotes their apoptosis by preventing the recruitment, and fusion of pre-osteoclasts, to keep osteoclasts away from the bone surface [29]. Some studies have shown that ALN can increase the degree of mineralization in bone by promoting the combination of hydroxyapatite crystals on the bone remodeling site [30]. Our results show that ALN maintains the structure of the subchondral bone, reduces the effect of knee instability on LIF, and protects the articular cartilage. However, we also observed that long-term application of ALN may lead to excessive mineralization of bone. And clinically, the side effects of high-dose ALN would likely result in osteonecrosis [31]. In addition, ALN has no significant effect on the amount of sclerostin in osteocytes [32]. This also indirectly shows that ALN inhibits the number of osteoclasts and reduces the expression of the osteoclast-derived factor, LIF, thereby affecting the changes in sclerostin.

In conclusion, this study showed that subchondral bone remodeling increased in the early stage of $\mathrm{OA}$, and a large number of osteoclasts secreted LIF to negatively regulate the expression of sclerostin in osteocytes, and then upregulated WNT/ $\beta$-catenin to promote bone formation and restore coupled bone remodeling. The application of ALN to inhibit osteoclasts weakened the promotion of LIF on the early abnormal bone remodeling of OA and attenuated osteoarthritis. However, this study has certain limitations, and more in vitro and in vivo experiments are needed for further verification to determine the regulatory mechanism of LIF in abnormal bone remodeling. This study provides a deeper understanding of the role of clastokine LIF in abnormal bone remodeling in early OA.

\section{Abbreviations}

OA: Osteoarthritis; ALN: Alendronate; LIF: Leukemia inhibitory factor; SOST: Sclerostin; KO: Knockout; BV/TV: Bone volume/total tissue volume; SBP.Th: Subchondral bone plate thickness; Tb.Sp: Trabecular separation; HRQOL: 
Health-related quality of life; GWAS: Genome-wide association studies; M-CSF: Macrophage colony-stimulating factor; RANKL: Receptor activator for nuclear factor-KB ligand; ACLT: Anterior cruciate ligament transection; TRAP: Tartrateresistant acid phosphatase; MMP-13: Matrix metalloproteinase 13; Col X: Type $X$ collagen; TGF- $\beta$ : Transforming growth factor $\beta$; IGF-I: Insulin-like growth factor I; CHR: Cytokine binding region; gp130: Glycoprotein 130; CT-1: Cardiotrophin 1; OSM: Oncostatin M; LRP5: Lipoprotein receptor-related protein 5.

\section{Supplementary Information}

The online version contains supplementary material available at https://doi. org/10.1186/s12891-021-04886-2.

Additional file 1. The study was carried out in compliance with the ARRIVE guidelines.

\section{Acknowledgments}

We would like to express our sincere thanks to Prof. Hong Zhou from the ANZAC Institute of University of Sydney for the guidance and support for this study.

\section{Authors' contributions}

QJ designed the experiment. XZ, LM, HG, JW, LY conducted the main experiments. $X Z$ analyzed the data and was a major contributor in writing the manuscript. XZ, SZ, XY confirm the authenticity of all the raw data. All the authors read and approved the final submitted manuscript.

\section{Funding}

This study was supported by grants from the National Natural Science Foundation of China (No.81860400 and No.81660373) and Ningxia Key R\&D Project (2018BEG02005).

\section{Availability of data and materials}

The datasets used during the current study are available from the corresponding author on reasonable request.

\section{Declarations}

\section{Ethics approval and consent to participate}

The experimental procedures were carried out in accordance with the National Institutes of Health Guide for the care and Use of Laboratory Animals, and were approved by the medical research ethics committee of the General Hospital of Ningxia Medical University (Yinchuan, China) (protocol No. 2018-056).

\section{Consent for publication}

Not applicable.

\section{Competing interests}

No potential conflict of interest was reported by the authors.

\section{Author details}

'Department of Orthopedics, General Hospital of Ningxia Medical University, 804 Shengli Street, Yinchuan, Ningxia, China. ${ }^{2}$ School of Clinical Medicine, Ningxia Medical University, Yinchuan, Ningxia, China.

Received: 8 August 2021 Accepted: 17 November 2021

Published online: 25 January 2022

\section{References}

1. Xie Y, Yu Y, Wang JX, et al. Health-related quality of life and its influencing factors in Chinese with knee osteoarthritis. Qual Life Res. 2020;29:2395-402.

2. Lo J, Chan L, Flynn S. A Systematic Review of the Incidence, Prevalence, Costs, and Activity and Work Limitations of Amputation, Osteoarthritis, Rheumatoid Arthritis, Back Pain, Multiple Sclerosis, Spinal Cord Injury,
Stroke, and Traumatic Brain Injury in the United States: A 2019 Update. Arch Phys Med Rehabil. 2020;102(1):115-31.

3. Weber A, Chan PMB, Wen C. Do immune cells lead the way in subchondral bone disturbance in osteoarthritis? Prog Biophys Mol Biol. 2019;148:21-31.

4. Donell S. Subchondral bone remodelling in osteoarthritis. EFORT Open Rev. 2019:4:221-9.

5. Burr DB, Gallant MA. Bone remodelling in osteoarthritis. Nat Rev Rheumatol. 2012;8:665-73.

6. Dallas SL, Prideaux M, Bonewald LF. The osteocyte: an endocrine cell ... and more. Endocr Rev. 2013;34:658-90.

7. Ono T, Takayanagi H. Osteoimmunology in bone fracture healing. Curr Osteoporos Rep. 2017;15:367-75.

8. Poulton IJ, McGregor NE, Pompolo S, Walker EC, Sims NA. Contrasting roles of leukemia inhibitory factor in murine bone development and remodeling involve region-specific changes in vascularization. J Bone Miner Res. 2012;27:586-95.

9. Grimaud E, Blanchard F, Charrier C, Gouin F, Redini F, Heymann D. Leukaemia inhibitory factor (lif) is expressed in hypertrophic chondrocytes and vascular sprouts during osteogenesis. Cytokine. 2002;20:224-30.

10. Koide M, Kobayashi Y, Yamashita T, et al. Bone formation is coupled to resorption via suppression of Sclerostin expression by osteoclasts. J Bone Miner Res. 2017;32:2074-86.

11. Weivoda MM, Youssef SJ, Oursler MJ. Sclerostin expression and functions beyond the osteocyte. Bone. 2017;96:45-50.

12. Klein JC, Keith A, Rice SJ, et al. Functional testing of thousands of osteoarthritis-associated variants for regulatory activity. Nat Commun. 2019;10:2434.

13. Boyce BF. Advances in the regulation of osteoclasts and osteoclast functions. J Dent Res. 2013;92:860-7.

14. Henriksen K, Neutzsky-Wulff AV, Bonewald LF, Karsdal MA. Local communication on and within bone controls bone remodeling. Bone. 2009;44:1026-33.

15. Khorasani MS, Diko S, Hsia AW, et al. Effect of alendronate on posttraumatic osteoarthritis induced by anterior cruciate ligament rupture in mice. Arthritis Res Ther. 2015;17:30.

16. Intema F, Hazewinkel HA, Gouwens D, et al. In early OA, thinning of the subchondral plate is directly related to cartilage damage: results from a canine ACLT-meniscectomy model. Osteoarthr Cartil. 2010;18:691-8.

17. Bolbos Rl, Zuo J, Banerjee S, et al. Relationship between trabecular bone structure and articular cartilage morphology and relaxation times in early OA of the knee joint using parallel MRI at 3 T. Osteoarthr Cartil. 2008;16:1150-9.

18. Neogi T, Felson D, Niu J, et al. Cartilage loss occurs in the same subregions as subchondral bone attrition: a within-knee subregion-matched approach from the multicenter osteoarthritis study. Arthritis Rheum. 2009;61:1539-44

19. Neuhold LA, Killar L, Zhao W, et al. Postnatal expression in hyaline cartilage of constitutively active human collagenase-3 (MMP-13) induces osteoarthritis in mice. J Clin Invest. 2001;107:35-44.

20. von der Mark K, Kirsch T, Nerlich A, et al. Type X collagen synthesis in human osteoarthritic cartilage. Indication of chondrocyte hypertrophy. Arthritis Rheum. 1992:35:806-11.

21. Tang $Y$, Wu X, Lei W, et al. TGF-beta1-induced migration of bone mesenchymal stem cells couples bone resorption with formation. Nat Med. 2009;15:757-65.

22. Xian $L$, Wu X, Pang $L$, et al. Matrix IGF-1 maintains bone mass by activation of mTOR in mesenchymal stem cells. Nat Med. 2012;18:1095-101.

23. Ota K, Quint $P$, Weivoda MM, et al. Transforming growth factor beta 1 induces CXCL16 and leukemia inhibitory factor expression in osteoclasts to modulate migration of osteoblast progenitors. Bone. 2013;57:68-75.

24. Boulanger MJ, Bankovich AJ, Kortemme T, Baker D, Garcia KC. Convergent mechanisms for recognition of divergent cytokines by the shared signaling receptor gp130. Mol Cell. 2003;12:577-89.

25. Walker EC, McGregor NE, Poulton IJ, et al. Oncostatin M promotes bone formation independently of resorption when signaling through leukemia inhibitory factor receptor in mice. J Clin Invest. 2010;120:582-92.

26. Ellies DL, Viviano B, McCarthy J, et al. Bone density ligand, Sclerostin, directly interacts with LRP5 but not LRP5G171V to modulate Wnt activity. J Bone Miner Res. 2006;21:1738-49. 
27. Wu L, Guo H, Sun K, Zhao X, Ma T, Jin Q. Sclerostin expression in the subchondral bone of patients with knee osteoarthritis. Int J Mol Med. 2016;38:1395-402.

28. Li J, Xue J, Jing Y, et al. SOST deficiency aggravates osteoarthritis in mice by promoting sclerosis of subchondral bone. Biomed Res Int. 2019;2019:7623562

29. Mulcahy LE, Curtin CM, McCoy RJ, et al. The effect of bisphosphonate treatment on the biochemical and cellular events during bone remodelling in response to microinjury stimulation. Eur Cell Mater. 2015;30:271-81.

30. Spadaro JA, Damron TA, Horton JA, et al. Density and structural changes in the bone of growing rats after weekly alendronate administration with and without a methotrexate challenge. J Orthop Res. 2006;24:936-44.

31. Diel IJ, Bergner R, Grotz KA. Adverse effects of bisphosphonates: current issues. J Support Oncol. 2007;5:475-82.

32. Macias BR, Swift JM, Nilsson MI, Hogan HA, Bouse SD, Bloomfield SA. Simulated resistance training, but not alendronate, increases cortical bone formation and suppresses sclerostin during disuse. J Appl Physiol. 2012;112:918-25.

\section{Publisher's Note}

Springer Nature remains neutral with regard to jurisdictional claims in published maps and institutional affiliations.

- fast, convenient online submission

- thorough peer review by experienced researchers in your field

- rapid publication on acceptance

- support for research data, including large and complex data types

- gold Open Access which fosters wider collaboration and increased citations

- maximum visibility for your research: over 100M website views per year

At BMC, research is always in progress.

Learn more biomedcentral.com/submissions 